\title{
Stochastics Calculus: Malliavin Calculus in a simplest way
}

\author{
${ }^{1}$ Udoye, Adaobi Mmachukwu, ${ }^{2 .}$ Akoh, David' ${ }^{3 \cdot}$ Olaleye, Gabriel C. \\ Department of Mathematics, University of Ibadan, Ibadan. \\ Department of Mathematics, Federal Polytechnic, Bida \\ Department of Mathematics, Federal Polytechnic, Bida.
}

\begin{abstract}
We present the theory of Malliavin Calculus by tracing the origin of this calculus as well as giving a simple introduction to the classical variational problem. In the work, we apply the method of integration-byparts technique which lies at the core of the theory of stochastic calculus of variation as provided in Malliavin Calculus. We consider the application of this calculus to the computation of Greeks, as well as discussing the calculation of Greeks (price sensitivities) by considering a one dimensional Black-Scholes Model. The result shows that Malliavin Calculus is an important tool which provides a simple way of calculating sensitivities of financial derivatives to change in its underlying parameters such as Delta, Vega, Gamma, Rho and Theta.
\end{abstract}

\section{Introduction}

The Malliavin Calculus also known as Stochastic Calculus of Variation was first introduced by Paul Malliavin as an infinite-dimensional integration by parts technique. This calculus was designed to prove results about the smoothness of densities of solutions of stochastic differential equations driven by Brownian motion. Malliavin developed the notion of derivatives of Wiener functional as part of a programme for producing a probabilistic proof of the celebrated Hörmander theorem, which states that solutions to certain stochastic differential equations have smooth transition densities.

Classical variational problems are problems that deal with selection of path from a given family of admissible paths in order to minimize the value of some functionals. The calculus of variation originated with attempts to solve Dido's problem known as the isoperimetric problem. An infinite dimensional differential calculus on the Wiener space, known as Malliavin Calculus, was initiated by Paul Malliavin (1976) with the initial goal of giving conditions insuring that the law of a random variable has a density with respect to Lebesgue measure, as well as estimates for this density and its derivative. Malliavin Calculus looks forward to finding the derivative of the functions of Brownian motion which will be referred to as Malliavin derivative. We will highlight the theory of Malliavin Calculus. In what follows, $\mathrm{H}$ is a real separable Hilbert space with inner product $\langle., .\rangle_{\nexists} . \Omega$ denotes the sample space, $\mathrm{P}$ denotes the probability space $\mathrm{P}$.

\section{The Wiener Chaos Decomposition}

Definition 2.1. A stochastic process $\mathrm{W}=\{\mathrm{W}(\mathrm{h}), \mathrm{h} \in \mathrm{H}\}$ defined in a complete probability space $(\Omega, F, P)$ is called an isonormal Gaussian process if $\mathrm{W}$ is a centered Gaussian family such that

$$
\mathrm{E}(\mathrm{W}(\mathrm{h}) \mathrm{W}(\mathrm{g}))=\langle h, g\rangle_{\mathbb{Z}} \text { for all } \mathrm{h}, \mathrm{g} \in \mathrm{H} \text {. }
$$

Remark 2.2. The mapping $\mathrm{h} \rightarrow \mathrm{W}(\mathrm{h})$ is linear [8]. From the above, we have that $\|W(h)\|_{L^{2}(P)}^{2}=\mathrm{E}\left(W(h)^{2}\right)=\|h\|^{2} \sharp$. Let $\mathrm{G}$ be the $\sigma$-field generated by the random variables $\{\mathrm{W}(\mathrm{h}), \mathrm{h} \in \mathrm{H}\}$, the main objective of this part is to find a decomposition of $L^{2}(\Omega, G, P)$. We state some results concerning the Hermite polynomials in order to find the decomposition .

Let $\mathrm{H}_{n}(x)$ denote the nth Hermite polynomial, then

$$
\mathrm{H}_{n}(x)=\frac{(-1)^{n}}{n !} e^{\frac{x^{2}}{2}} \frac{d^{n}}{d x^{n}}\left(e^{-\frac{x^{2}}{2}}\right), \mathrm{n} \geq 1
$$

and $\mathrm{H}_{0}(x)=1$. These hermite polynomials are coefficients of the power expansion in $\mathrm{t}$ of the function $F(t, x)=\exp \left(t x-\frac{t^{2}}{2}\right)$ which can easily be seen by rewriting

$$
F(t, x)=\exp \left(\frac{x^{2}}{2}-\frac{1}{2}(x-t)^{2}\right)
$$

and expanding the function around $\mathrm{t}=0$. 
The power expansion combines with some particular properties of $\mathrm{F}$, that is

$$
\begin{aligned}
& \frac{\partial F}{\partial x}=t \exp \left(t x-\frac{t^{2}}{2}\right)=t F \\
& \frac{\partial F}{\partial t}=(x-t) \exp \left(t x-\frac{t^{2}}{2}\right)=(x-t) F \\
& F(-x, t)=\exp \left(-t x-\frac{t^{2}}{2}\right)=F(x,-t)
\end{aligned}
$$

provides the corresponding properties of the Hermite polynomials for $\mathrm{n} \geq 1$

- $\quad H_{n}^{\prime}(x)=H_{n-1}(x)$

- $\quad(n+1) H_{n+1}(x)=x H_{n}(x)-H_{n-1}(x)$

- $H_{n}(-x)=(-1)^{n} H_{n}(x)$

This is shown by using induction method:

To show that $H_{n}^{\prime}(x)=H_{n-1}(x)$;

Let $\mathrm{n}=1$, from

$$
\mathrm{H}_{n}(x)=\frac{(-1)^{n}}{n !} e^{\frac{x^{2}}{2}} \frac{d^{n}}{d x^{n}}\left(e^{-\frac{x^{2}}{2}}\right)
$$

we have

$$
\begin{gathered}
H_{1}^{\prime}(x)=\left(-e^{\frac{x^{2}}{2}} \frac{d}{d x}\left(e^{-\frac{x^{2}}{2}}\right)\right)^{\prime}=\left(-e^{\frac{x^{2}}{2}}(-x) e^{-\frac{x^{2}}{2}}\right)^{\prime} \\
=x^{\prime}=1=H_{n-1}=H_{0}(x)
\end{gathered}
$$

Let $\mathrm{n}=2$,

$$
\begin{aligned}
H_{2}^{\prime}(x) & =\left[\frac{1}{2} e^{\frac{x^{2}}{2}} \frac{d^{2}}{d x^{2}}\left(e^{-\frac{x^{2}}{2}}\right)\right]^{\prime}=\left[\frac{1}{2} e^{\frac{x^{2}}{2}} \frac{d}{d x}\left(-x e^{-\frac{x^{2}}{2}}\right)\right]^{\prime} \\
& =\left[\frac{1}{2} e^{\frac{x^{2}}{2}}\left(-e^{-\frac{x^{2}}{2}}+x^{2} e^{-\frac{x^{2}}{2}}\right)\right]^{\prime}=\frac{1}{2}\left(-1+x^{2}\right)^{\prime}=x=H_{1}(x) .
\end{aligned}
$$

Also for $\mathrm{n}=3$ we have

$$
H_{3}^{\prime}(x)=\left[-\frac{1}{6} e^{\frac{x^{2}}{2}} \frac{d^{3}}{d x^{3}}\left(e^{-\frac{x^{2}}{2}}\right)\right]^{\prime}=-\frac{1}{6}\left[x+2 x-x^{3}\right]=\frac{1}{2}\left(x^{2}-1\right)=H_{2}(x) \text {. }
$$

Lemma 2.3. Let $X, Y$ be two random variables with joint Gaussian distribution such that $\mathrm{E}(\mathrm{X})=\mathrm{E}(\mathrm{Y})=0$ and $\mathrm{E}\left(\mathrm{X}^{2}\right)=\mathrm{E}\left(\mathrm{Y}^{2}\right)=1$. Then for all $\mathrm{m}, \mathrm{n} \geq 0$, we have

$$
\mathrm{E}\left(H_{n}(X) H_{m}(Y)\right)= \begin{cases}0, & \text { if } \mathrm{n} \neq \mathrm{m} ; \\ \frac{1}{\mathrm{n} !}\left(\mathrm{E}(X Y)^{\mathrm{n}}\right), & \text { if } \mathrm{n}=\mathrm{m},\end{cases}
$$

Proof. See the proof of lemma 1.1.1 in [8] 
Lemma 2.4 The random variables $\left\{\mathrm{e}^{\mathrm{w}(\mathrm{h})}, h \in \mathbb{Z}\right\}$ form a totalsubset of $L^{2}(\mathfrak{G})=$ $L^{2}(\Omega, \mathfrak{g}, P)$.

Proof. Let $X \in L^{2}(\mathfrak{g})$ such that $\mathrm{E}\left(X e^{W(h)}\right)=0$ for all $h \in \mathbb{Z}[9]$. By thelinearity of mapping $h \rightarrow W(h)$ we have

$$
\mathrm{E}\left(X \exp \left(\sum_{i=1}^{m} t_{1} W(h)\right)\right)=0, t_{i} \in \mathbb{R}, h_{i} \in \mathbb{Z}, i=1,2, \ldots m, m \geq 1
$$

Equation (2) shows that theLaplace of $v$ is given by

$$
v(\mathrm{~B})=\mathrm{E}\left(X_{1 B}\left(W\left(h_{1}\right), \ldots, W\left(h_{m}\right)\right)\right)
$$

for a Borel set $\mathcal{Z} \in \mathrm{R}^{m}$. As the transformis zero, the measure $v$ must be zero for every set $\mathrm{G} \in \mathcal{G}$. That is, $\mathrm{E}\left(X_{1 G}\right)=0$ for $\mathrm{G} \in \mathcal{G} \Rightarrow X=0$.

Definition2.5. For each $n \geq 1$, we define $\mathbb{Z}_{n}$ the choas of order $\mathrm{n}$ as the closed linear subspace of $L^{2}(\Omega, F, P)$ generated by therandom variables $\left\{H_{n}(W(h)): h \in \mathbb{Z},\|h\|_{\mathbb{Z}}=1\right.$. Theorem 2.6. The space $L^{2}(\Omega, \mathcal{G}, P)$ can be decomposed into the infinity orthogonal sum of the subspaces $\mathbb{Z}_{n}[9]$ :

$$
L^{2}(\Omega, \mathfrak{g}, P)=\bigoplus_{n=0}^{\infty} \mathbb{H}_{n} .
$$

proof.Let $X \in L^{2}(\Omega, F, P)$ be orthogonal to $\mathbb{H}_{n}$ for all $\mathrm{n} \geq 0$. We show that $X=0$.

We have that $\mathrm{E}\left(X H_{n}(W(h))\right)=0$ for all $h \in \mathcal{H}^{*}$ such that $/ h / /_{\mathbb{Z}}=1$. Using the fact that $x^{n}$ can be expressed as a linear combination of theHermite polynomial $H_{r}(x), 0 \leq r \leq n$, we have $\mathrm{E}\left(X W(h)^{n}\right)=0 \forall n \geq 0$ therefore,

$$
\mathrm{E}(X \exp (t W(h)))=0
$$

$\forall t \in \mathrm{R}$ and for all $\mathrm{h} \in \mathcal{Z}^{*}$ of norm 1 . By thelemma above which states that the random variables $\left\{\mathrm{e}^{\mathrm{W}(\mathrm{h})}, h \in \mathbb{Z}\right\}$ form a totalsubset of $L^{2}(\Omega, \mathcal{G}, P)$, we have that equation (3) implies that $\mathrm{X}=0$

\section{The Malliavin Derivative}

We consider the set $\mathrm{C}_{p}^{\infty}\left(\mathrm{R}^{n}\right)$ of all infinitely differentiable functions $f: \mathrm{R}^{n} \rightarrow \mathrm{R}$ denoted such that $f$ and all of its derivatives have at most poly nomialgrowth ( $p$ denoted partial derivatives with polynomial growth). Let $\mathrm{n} \geq 1$ and $f \in \mathrm{C}_{p}^{\infty}\left(\mathrm{R}^{n}\right)$, we denote by $S$ the set of all random variable the form

$$
F=f\left(W\left(h_{1}\right), \ldots, W\left(h_{n}\right)\right)
$$

where $h_{1}, \ldots, h_{n} \in \mathcal{H}$ and $F \in S$, a smooth variable.

Definition3.1. The derivative of random variable $F \in S$ is define as

$$
\mathrm{DF}=\sum_{\mathrm{i}=\mathrm{o}}^{n} \partial_{\mathrm{i}} f\left(W\left(h_{1}\right), \ldots, W\left(h_{n}\right)\right) h_{i} .
$$

where the derivative is a mapping DF: $\Omega \rightarrow \mathcal{Z}$. 
Lemma 3.2 Let $F \in \mathcal{Z}$ and $h \in \mathbb{Z}$ then

$$
\mathrm{E}\left(\langle D F, h\rangle_{\mathbb{Z}}\right)=\mathrm{E}(F W(h)) \text {. }
$$

Proof. Suppose that $\|h\|_{\mathcal{Z}}=1$. Due to the linearity of the scalar product on $\mathrm{W}$, there exists an orthonomal family of $\mathcal{Z},\left\{e_{1}, \ldots e_{n}\right\}$ such that $h=e_{1}$, such that that we can write $F$ as

$$
F=f\left(W\left(e_{1}\right), \ldots, W\left(e_{n}\right)\right)
$$

for a suitable function $f \in \mathrm{C}_{p}^{\infty}\left(\mathrm{R}^{n}\right)$. Let $\Psi(x)$ be the multivariate density of the standard normal distribution, that is

$$
\psi(x)=(2 \pi)^{-\frac{n}{2}} \exp \left(-\frac{1}{2} \sum_{i=1}^{n} x_{i}^{2}\right)
$$

We have by classical integration by partsformular

$$
\begin{aligned}
\mathrm{E}\left(\langle D F, h\rangle_{\sharp}\right) & =\int_{\mathbb{R}^{n}} \partial_{1} f(x) \psi(x) x_{1} d x=\int_{\mathbb{R}^{n}} f(x) \psi(x) x d x \\
& =\mathrm{E}\left(F W\left(e_{1}\right)\right)=\mathrm{E}(F W(h)) .
\end{aligned}
$$

This completes the proof.

Proposition 3.3. (Nualart,2006). Let $\varphi: \mathrm{R}^{m} \rightarrow \mathrm{R}$ be a continously diferentiable function with bounded partial derivatives. Suppose $F=\left(F_{1}, \ldots, F_{m}\right)$ is a random vector with componentsin $\mathrm{D}^{1, p}$. Then $\varphi(F)$ is in $\mathrm{D}^{1, p}$ and

$$
D(\varphi(F))=\sum_{i=1}^{m} \partial_{i} \varphi(F) D F^{i}
$$

The proof of proposition 3.3 is similar to the proof of proposition 1.20, pp. 13

Of (Nualart, 2009). The chain rule can be extended to the case of a Lipschitz function.

Theorem 3.4 (Closability).

Assume $F \in L^{2}(P)$ and $\mathrm{F}_{\mathrm{k}} \in \mathrm{D}^{1,2}, k=1,2, \ldots$ such that

1. $F_{k} \rightarrow F, k \rightarrow \infty$, in $L^{2}(P)$

2. $\left\{D_{t} F_{k}\right\}_{k=1}^{\infty}$ converges in $\mathrm{L}^{2}(P \times \lambda)$.

Then $\mathrm{F} \in \mathrm{D}^{1,2}$ and $D_{t} F_{k} \rightarrow D_{t} F, k \rightarrow \infty$, in $\mathrm{L}^{2}(P \times \lambda)$.

Proof. Let $F=\sum_{n=0}^{\infty} I_{n}\left(f_{n}\right)$ and $F_{k} \sum_{n=0}^{\infty} I_{n}\left(f_{n}^{(k)}\right), k=1,2, \ldots$.

By (1) we have $f_{n}^{(k)} \rightarrow f_{n}, k \rightarrow \infty$, in $L^{2}\left(\lambda^{n}\right)$ for all $\mathrm{n}$.

By (2) we have

$$
\sum_{n=1}^{\infty} n n !\left\|f_{n}^{(k)}-f_{n}^{(j)}\right\|_{L^{2}\left(\lambda^{n}\right)}^{2}=\left\|D_{t} F_{k}-D_{t} F_{j}\right\|_{L^{2}(P \times \lambda)}^{2} \rightarrow 0, j, k \rightarrow \infty
$$

Hence, by Fatou Lemma,

$$
\lim _{k \rightarrow \infty} \sum_{n=1}^{\infty} n n !\left\|f_{n}^{(k)}-f_{n}\right\|_{L^{2}\left(\lambda^{n}\right)}^{2} \leq \lim _{k \rightarrow \infty} \lim _{j \rightarrow \infty} \sum_{n=1}^{\infty} n n !\left\|f_{n}^{(k)}-f_{n}^{(j)}\right\|_{L^{2}\left(\lambda^{n}\right)}^{2}=0 .
$$

This gives $F \in \mathrm{D}^{1,2}, D_{t} F_{k} \rightarrow D_{t} F, k \rightarrow \infty$, in $L^{2}(P \times \lambda)$.

Proposition 3.5. [8] Suppose $F \in \mathrm{D}^{1,2}$ is a square integrable random variable With a decomposition given above, we have 


$$
D_{t} F=\sum_{n=1}^{\infty} n I_{n-1}\left(f_{n}(., t)\right) .
$$

Proof. We prove the statement for a simple function (see proposition 1.2.7 of [8]).

Using a simple function, the general case results from the density of the simple function.

Let $F=I_{m}\left(f_{m}\right)$ for a symmetric function $f_{m}$, then, by applying

$$
D F=\sum_{i=0}^{n} \partial_{i} f\left(W\left(h_{1}\right), \ldots, W\left(h_{n}\right)\right) h_{i}
$$

to $g\left(W\left(A_{i 1}\right), \ldots, W\left(A_{i n}\right)\right)$ with $g\left(x_{1}, \ldots, x_{n}\right)=x_{1}, \ldots, x_{n}$ we have

$$
D_{t} F=\sum_{j=1}^{m} \sum_{i 1, \ldots, j m=1}^{n} a_{i 1 \ldots i m} W\left(A_{i 1}\right) \ldots 1_{A i j} \ldots W\left(A_{i m}\right)=m I_{m-1}\left(f_{m}(., t)\right) \text {. }
$$

Theorem 3.6. Let $\mathrm{F}$ be a square integrable random variable denoted by

$$
F=\sum_{n=0}^{\infty} I_{n}\left(f_{n}\right) \text {. Let } A \in \mathrm{B} \text {, then } \mathrm{E}\left(\mathrm{F} \backslash \mathcal{F}_{A}\right)=\sum_{n=0}^{\infty} I_{n}\left(f_{n} 1_{A}^{\otimes_{n}}\right) \text {. }
$$

Proof. Assume that $F=I_{n}\left(f_{n}\right)$ such that $f_{n}$ is a function in $E_{n}$. We also

assume that the kernel $f_{n}$ is of the form $1_{B_{1} \times \ldots \times B_{n}}$ with $B_{1}, \ldots, B_{n}$ being mutually

disjoint sets of finite measure. Through the linearity of $\mathrm{W}$ and the properties of the conditional expectation we have

$$
\begin{aligned}
& E\left(F \backslash \mathcal{Z}_{A}\right)=E\left(W\left(B_{1}\right) \ldots W\left(B_{n}\right) / \mathcal{Z}_{A}\right)=E\left(\prod_{i=1}^{n}\left(W\left(B_{i} \cap A\right)+W\left(B_{i} \cap A^{c}\right)\right) \backslash \mathcal{Z}_{A}\right) \\
& =I_{n}\left(1_{\left(B_{1} \cap A\right) \times . . \times\left(B_{n} \cap A\right)}\right) .
\end{aligned}
$$

\section{The Divergence Operator}

In this section, we consider the divergence operator, defined as the adjoint of the derivative operator. If the underlying Hilbert $\mathbb{Z}$ is an $L^{2}$-space of the form $L^{2}(T, \mathcal{Z}, \mu)$, where $\mu$ is a $\quad \sigma$-finit eatomless measure, we interpret the divergence operator as a stochastic integral and call it Skorohod integral because in the Brownian motion case it coincides with the generalization of the ItÔ stochastic integral to anticipating integrands. We first introduce the divergence operator in the framework of Gaussian isonormal process $W=\{W(h), h \in \mathbb{Z}\}$ associated with the Hilbert space $\mathbb{Z}$. We assume that $\mathrm{W}$ is defined on a complete probability space $(\Omega, \mathcal{Z}, P)$, and that $\mathcal{Z}$ is generated by W. We shall consider results from[1], [8] [ and [12]. We note that the derivative operator $D$ is a closed and unbounded operator with values in $L^{2}(\Omega ; \not{Z})$ defined on the dense subset $\mathrm{D}^{1,2}$ of $L^{2}(\Omega)$.

Definition 4.1. The adjoint of the operator $D$ denote by $\delta$ is an unbounded operator on $L^{2}(\Omega ; \not)$ and satisfies

- The domain of $\delta$ denoted as Dom $\delta$ is the set of $\not$-valued square integrable random variable $u \in L^{2}(\Omega, \not{Z})$ where

$$
\left|\mathrm{E}\left(\langle D F, u\rangle_{\sharp}\right)\right| \leq c\|F\|_{2} \quad \text { for all } F \in \mathrm{D}^{1,2}
$$

such that the $\mathrm{c}$ is a constant depending on $\mathrm{u}$.

- $\quad$ Let $\mathrm{u} \in \operatorname{Dom} \delta$, then $\delta(\mathrm{u})$ is an element of $L^{2}(\Omega)$ characterized by

$$
\mathrm{E}(F \delta(u))=\mathrm{E}\left(\langle D F, u\rangle_{\#}\right)
$$

each for $F \in \mathrm{D}^{1,2}$.

From equation (8) above, $\mathrm{E}(\delta(\mathrm{u}))=0$ if $\mathrm{u} \in \mathrm{Dom} \delta$ and $\mathrm{F}=1$. We see $\mathrm{S}_{\mathrm{H}}$ to be the class of smooth elementary elements of the form

$$
u=\sum_{j=1}^{n} F_{j} h_{j}
$$


such that $F_{j}$ are smooth random variables and the $h_{j}$ are elements of $\#$. Applying integration-by-parts formula, we deduce that $\mathrm{u} \epsilon \operatorname{Dom} \delta$ and

$$
\delta(u)=\sum_{j=1}^{n} F_{j} W\left(h_{j}\right)-\sum_{j=1}\left\langle D F_{j} h_{j}\right\rangle_{\sharp}
$$

Theorem 4.2. Let $\mathrm{F} \in \mathrm{D}^{1,2}$ and $\mathrm{u} \in$ Dom $\delta$ such that $F u \in L^{2}(\Omega ; \not{Z})$. Then Fu belongs to Dom $\delta$ and we have the equality

$$
\delta(F u)=F \delta(u)-\langle D F, u\rangle_{\#}
$$

provided the right hand side is square integrable.

Proof. Let $\mathrm{G}$ be any smooth random variable, we obtain

$$
\begin{gathered}
\mathrm{E}[G \delta(F u)]=\mathrm{E}\left[\langle D G, F u\rangle_{\mathbb{Z}}\right]=\mathrm{E}\left[\langle u, D(F G)-G D F\rangle_{\mathbb{Z}}\right\rfloor \\
=\mathrm{E}\left[\left(\delta(u) F-\langle u, D F\rangle_{\mathbb{Z}}\right) G\right]
\end{gathered}
$$

Lemma 4.3. Let $D^{h}(u):=\sum_{j=1}^{n} D^{h}\left(F_{j}\right) h_{j}$ where $u \in S_{\nexists \mathbb{H}}$, the class of smooth elementary processes of the form $u=\sum_{j=1}^{n} F_{j} h_{j}, F \in S, h \in \mathbb{Z}$, we have that the following commutativity relationship holds

$$
D^{h}(\delta(u))=\langle u, h\rangle_{\sharp}+\delta\left(D^{h} u\right)
$$

This is true from the following:

$$
\delta(u)=\sum_{j=1}^{n} F_{j} W\left(h_{j}\right)-\sum_{j=1}\left\langle D F_{j} h_{j}\right\rangle_{\nexists} .
$$

yields

$$
\begin{aligned}
D^{h}(\delta(u)) & =\sum_{j=1}^{n}\left\langle D\left(F_{j} W\left(h_{j}\right)\right)-D\left\langle D F_{j}, h_{j}\right\rangle_{\mathbb{Z}}, h\right\rangle_{\mathbb{Z}} \\
= & \sum_{j=1}^{n} F_{j}\left\langle h, h_{j}\right\rangle_{\mathbb{Z}}+\sum_{j=1}^{n}\left(D^{h} F_{j} W\left(h_{j}\right)-\left\langle D\left(D^{h} F_{j}\right), h_{j}\right\rangle_{\mathbb{Z}}\right) \\
= & \langle u, h\rangle_{\mathbb{Z}}+\delta\left(D^{h} u\right) .
\end{aligned}
$$

Remark 4.4. Let $\boldsymbol{h} \in \mathcal{Z}$ and $F \in \mathrm{D}^{\mathrm{h}, 2}$. Then $F h$ belongs to domain of $\delta$ and the is true

$$
\delta(F h)=F W(h)-D^{h} F .
$$

\section{Theorem 4.5 (The Clark-Ocone formula).}

Let $F \in \mathrm{D}^{1,2}$ and $\mathrm{W}$ is a one-dimensional Brownian motion on [0,1]. Then

$$
F=\mathrm{E}(F)+\int_{0}^{T} E\left(D_{t} F \mid \mathcal{F}_{t}\right) d W_{t} .
$$

Proof. Suppose that $F=\sum_{n=0}^{\infty} I_{n}\left(f_{n}\right), n=1,2, \ldots$ (see[9] and Proposition 1.3.8 of

[12], we have

$$
\begin{gathered}
\int_{0}^{T} E\left[D_{t} F \mid \mathcal{Z}_{t}\right]=\int_{0}^{T} E\left[\sum_{n=1}^{\infty} n I_{n-1}\left(f_{n}(., t)\right) \mid \boldsymbol{\exists}_{t}\right] d W(t) \\
=\int_{0}^{T} \sum_{n=1}^{\infty} n E\left[I_{n-1}\left(f_{n}(., t)\right) \mid \boldsymbol{Z}_{t}\right] d W(t)
\end{gathered}
$$

Thus, 


$$
\begin{aligned}
& \int_{0}^{T} E\left[D_{t} F \mid \boldsymbol{F}_{t}\right]=\int_{0}^{T} \sum_{n=1}^{\infty} n I_{n-1}\left[f_{n}(., t) \cdot \boldsymbol{X}_{[0 . t]}^{\otimes}(.)\right] d W(t) \\
& =\int_{0}^{T} \sum_{n=1}^{\infty} n(n-1) ! \eta_{n-1}\left[f_{n}(., t) \boldsymbol{X}_{[0 . t]}^{\otimes(n-t)}\right] d W(t)=\sum_{n=1}^{\infty} n ! \eta_{n}\left[f_{n}(.)\right]=\sum_{n=1}^{\infty} I_{n}\left[f_{n}\right] \\
& =\sum_{n=1}^{\infty} I_{n}\left[f_{n}\right]-I_{0}\left[f_{0}\right]=F-\mathrm{E}[\mathrm{F}]
\end{aligned}
$$

Note. The Clark-Ocone formula and its proof can be found in [7], [8] and [10].

\section{A model of a financial market}

The Black-Scholes Model. We consider a market consisting of a non-risky asset (Bank account) $B$ and a risky asset (stock) $S$. Let the process of the risky asset be given by

$$
S(t)=S_{0} \exp \left(\left(\mu-\frac{\delta^{2}}{2}\right) t+\sigma W(t)\right)
$$

where $W=\{W(t): t \in[0, T]\}$ is a Brownian motion defined on a complete probabilityspace $(\Omega, \mathcal{Z}, P)$ and $\left\{\mathcal{Z}_{t}, t \in[0, T]\right\}$ is a filtration generated by Brownian motion $\sigma_{t}$ denote the volatility process, $\mu$ is the mean rate of return, and are all assumed to be constant.

The price of the bond $B(t)$ and the price of the stock $S(t)$ satisfies the differential equations:

and

$$
\begin{aligned}
& d B(t)=r B(t) d t \\
& \mathrm{~B}(0)=1
\end{aligned}
$$

$$
\begin{aligned}
& d S(t)=S(t)(\mu d t+\sigma d W(t)) \\
& \mathrm{S}(0)=0, \mathrm{~S}(\mathrm{t})=\mathrm{S}_{\mathrm{t}}
\end{aligned}
$$

We have that

$$
B(t)=B_{t}=e^{r t}
$$

where $r$ denotes the interest rate and it is a nonnegative adapted process satisfying

$$
\int_{0}^{T} r_{t} d t<\infty \text { a.s. }
$$

Definition 5.1. Let $Q$ be a probability measure on $(\Omega, 7)$ which is equivalent to $P$. Q is called equivalent (Local) martingale measure (or a non-risky probability measure) if the discounted price process $S_{t}=B_{t}^{-1} S_{t}=e^{-r t} S_{t}, t \in[0, T]$ is a local martingale under Q.

We note that: A process $X$ is sub-martingale (respectively, a super-martingale)

if and only if $X_{t}=M_{t}+A_{t}$ (respectively, $X_{t}=M_{t}-A_{t}$ ) where, $M$ is a local martingale and $\mathrm{A}$ is an increasing predictable process.

Suppose $\sigma_{t}>0$ for all $t \in[0, T]$ and $\int_{0}^{T}\left\|\theta_{\mathrm{s}}\right\|^{2} d s<\infty$ a.s.

where $\theta=\frac{\mu_{i}-F_{i}}{\sigma_{i}}$. We define the process

which is positive local martingale. If

$$
Z_{t}=\exp \left(-\int_{0}^{t} \theta_{s} d W_{s}-\frac{1}{2} \int_{0}^{t}\left\|\theta_{s}\right\|^{2} d s\right)
$$

$$
\mathrm{E}\left(\exp \left(-\int_{0}^{T} \theta_{s} d W_{s}-\frac{1}{2} \int_{0}^{T}\left\|\theta_{s}\right\|^{2} d t\right)\right)=1
$$

then the process $\mathrm{Z}_{\mathrm{T}}$ is a martingale and measure $\mathrm{Q}$ such that $\frac{d Q}{d P}=Z_{T}$ is a probability measure, equivalent to $\mathrm{P}$, such that under $\mathrm{Q}$, the process

$$
\tilde{W}_{t}=W_{t}+\int_{0}^{t} \theta_{s} d s
$$

is a Brownian motion.

In terms of the process $\tilde{W}_{t}$, the price process can be expressed as 


$$
S_{t}=S_{0} \exp \left(\int_{0}^{t}\left(r_{s}-\frac{\sigma^{2}}{2}\right) d s+\int_{0}^{t} \sigma_{s} d \tilde{W}\right)
$$

Thus, the discounted prices from a local martingale:

$$
\tilde{S}_{t}=B_{t}^{-1} S_{t}=S_{0} \exp \left(\int_{0}^{t} \sigma_{s} d \tilde{W}_{s}-\frac{1}{2} \int_{0}^{t} \sigma_{s}^{2} d s\right) .
$$

Definition 5.2. A derivative is contract on the risky asset that produces a payoff

$H$ at maturity $T$. The payoff is an $\boldsymbol{Z}_{T}$-measurable nonnegative random variable $H$.

Some fact about filtration:

- $\quad$ Filtrations are used to model the flow of information over time.

- $\quad$ At time $t$, one can decide if the event $\mathrm{A} \in \mathcal{Z}_{t}$ has occurred or not.

- Taking conditional expectation $E\left[X \mid \mathcal{F}_{T}\right]$ of a random variable $X$ means taking the expectation on the basis of all information available at time $t$.

\section{Proposition 5.3. (Girsanov theorem)}

There exist a probability $Q$ absolutely continuous with respect to $P$ such that $Q \circ T^{-1}=P\left(\right.$ that is, $\left.W_{t}+\int_{0}^{t} u_{s} d s\right)$ has the law of Brownian motion under $Q$ ) if and only if $\mathrm{E}\left(\xi_{1}\right)=1$ and in this case $\frac{d Q}{d P}=\xi_{1}$.

Proof. See Proposition 4.1.2, pp. 227 of [8]

Remark 5.4. The probability $P \circ T^{1}$ is absolutely continuous with respect to $P$.

Proposition 5.5 [8] Suppose that F, G are two random variables such that $F \in D^{1,2}$.

Let $u$ be an $\mathrm{H}$-valued random variable such that $\mathrm{D}^{\mathrm{u}} \mathrm{F}=\langle D F, u\rangle_{\mathbb{Z}} \neq 0$ almost surely and $\mathrm{Gu}\left(\mathrm{D}^{\mathrm{u}} \mathrm{F}\right)^{-1} \in \mathrm{Dom} \delta$. Then, for any differentiable function $f$ with bounded derivative we have

$$
\mathrm{E}\left(f^{\prime}(F) G\right)=\mathrm{E}(f(F) H(F, G))
$$

where

Proof. By chain rule, we have

$$
H(F, G)=\delta\left(G u\left(D^{u} F\right)^{-1}\right) .
$$

$$
D^{u}(f(F))=f^{\prime}(F) D^{u} F .
$$

By the duality relationship, equation (8), we obtain

$$
\begin{aligned}
\mathrm{E}\left(f^{\prime}(F) G\right) & =\mathrm{E}\left(D^{u}(f(F))\left(D^{u} F\right)^{-1} G\right)=\mathrm{E}\left(\left\langle D(f(F)), u\left(D^{u} F\right)^{-1} G\right\rangle_{\sharp}\right) \\
= & \mathrm{E}\left(f(F) \delta\left(G u\left(D^{u} F\right)^{-1}\right)\right)=\mathrm{E}\left(f(F) \delta\left(G u\left(D^{u} F\right)^{-1}\right)\right),
\end{aligned}
$$

We recall that in Malliavin Calculus, Integrating by part is

$$
\mathrm{E}\left[\int_{0}^{T}\left(D_{s} F\right) u(s) d s\right]=\mathrm{E}[F \delta(u)]=\mathrm{E}\left[F \int_{0}^{T} u(t) d W(t)\right] .
$$

\section{Applications}

Greeks are used for risk management purposes referred to as hedging in financial mathematics. Finite difference methods have been used to find the sensitivities of options by the use of Monte-Carlo methods, but the speed of convergence is not so fast very close to the discontinuities. The use of Malliavin Calculus provides a better way to calculate the greeks, both in terms of simplicity and speed of convergence. Thus, this method provides a good solution when the payoff function is strongly discontinuous. A greek can be defined as the derivative of a financial quantity with respect to a parameter of the model.

List of Greeks include:

- Delta: $=$ The derivative with respect to the price of the underlying; $\Delta:=\frac{\partial V}{\partial S}$. 
- Gamma: =Second derivative with respect to the price of the underlying;

$\Gamma:=\frac{\partial^{2} V}{\partial S^{2}}$

- Vega: $=$ Derivative with respect to the volatility; $v:=\frac{\partial V}{\partial \sigma}$

- $\quad$ Rho: $=$ Derivative with respect to interest rate; $\rho:=\frac{\partial V}{\partial r}$

- $\quad$ Theta: $=$ Derivative with respect to time; $\Theta:=\frac{\partial V}{\partial t}$

Greeks are useful in studying the stability of the quantity under variations of the chosen parameters. If the price of an option is calculated using the measure $Q$ as

$$
V=\mathrm{E}^{Q}\left[e^{-r(T-t)} \Phi(x)\right]
$$

where $\Phi(x)$ is the payoff function; the greek will be calculated under the same simulation together with the price. The equation gives

$$
\text { Greek }=\mathrm{E}^{Q}\left[e^{-r(T-t)} \Phi(x) \cdot \mathbf{W}\right]
$$

where $\mathrm{W}$ is a random variable called Malliavin weight.

Malliavin Calculus is a special tool for calculating sensitivities of financial derivatives to change in its underlying parameter. We now discuss the model of a financial market and the computation of the greeks.

\subsection{Computation of the Greeks}

We consider the Hilbert space which are constant on compact interval. We Assume that $W=\{W(h), h \in \mathrm{H}\}$ denotes an isonormal Gaussian process associated with the Hilbert space H. Let $W$ be defined on a complete probability space $(\Omega, \mathrm{F}, P)$ and let $\mathrm{F}$ be generated by $W$.

Remark 6.1.1 The following observation will be important for the application of proposition 5.5 .

- If $u$ is deterministic. Then, for $\mathrm{G} u\left(\mathrm{D}^{u} \mathrm{~F}\right)^{-1} \in \operatorname{Dom} \delta$ it suffices to say that

$\mathrm{G} u\left(\mathrm{D}^{u} \mathrm{~F}\right)^{-1} \in \mathrm{D}^{1,2}$ as this implies that $G u\left(\langle D F, u\rangle_{\sharp}\right)^{-1} \in \mathrm{D}^{1,2} \subset D o m \delta$.

- If $u=D F$, then the conclusion of Proposition 5.5 is written as

$$
\mathrm{E}\left[f^{\prime}(F) G\right]=\mathrm{E}\left[f(F) \delta\left(\frac{G D F}{\|D F\|_{\#}^{2}}\right)\right] \text {. }
$$

We consider an option with payoff $\mathrm{H}$ such that $\mathrm{E}^{\mathrm{Q}}\left(H^{2}\right)<\infty$. We have that

$V_{t}(\varphi)=E^{Q}\left(e^{-\int_{t}^{T} r_{s} d s} H \mid \boldsymbol{Z}_{t}\right)$. The price at $t=0$ gives $V_{0}=\mathrm{E}^{Q}\left(e^{-r T} H\right)$. Suppose

$\varsigma$ represent one of the parameters of $\mathrm{S}_{0}, \sigma, r$.

Let $H=f\left(F_{\varsigma}\right)$. Then

$$
\frac{\partial V_{0}}{\partial \varsigma}=e^{-r T} \mathrm{E}^{Q}\left(f^{\prime}\left(F_{\varsigma}\right) \frac{d F_{\varsigma}}{d \varsigma}\right)
$$

From Proposition 5.5, we have

$$
\frac{\partial \mathrm{V}_{0}}{\partial \varsigma}=e^{-r T} \mathrm{E}^{Q}\left(f\left(F_{\varsigma}\right) H\left(F_{\varsigma}, \frac{d F_{\varsigma}}{d \varsigma}\right)\right) .
$$

If $f$ is not smooth, then (14) provides better result in combination with Monte-Carlo Simulation than (13).

We note that the following:

- In the calculation of the greeks, differentiation can be done before finding the expectation, this will still give the same result.

- The Malliavin derivative of $D_{t} S_{T}=\frac{d S_{T}}{d W}(t, w)=\sigma S_{T}$.

- $\quad$ From proposition $5.5 D^{u} F$ must not be zero in order to make sure that $\frac{1}{D^{u} F}$

exist.

- $\quad$ Let $S(t)=S_{0} \exp \left(\left(\mu-\frac{\sigma^{2}}{2}\right) t+\sigma W(t)\right), t \in[0, T]$; then, 
$\frac{\partial S_{T}}{\partial S_{0}}=\exp \left(\left(\mu-\frac{\sigma^{2}}{2}\right) T+\sigma W(T)\right)=\frac{S_{T}}{S_{0}}$

- In calculating the greeks, we assume that

$S(t)=S_{0} \exp \left(\left(\mu-\frac{\sigma^{2}}{2}\right) t+\sigma W(t)\right), t \in[0, T]$

except otherwise stated.

\subsection{Computation of Delta}

We discuss delta of European options. Suppose $H$ depends only on the price of the stock at maturity time T, that is, $\mathrm{H}=\Phi\left(\mathrm{S}_{\mathrm{T}}\right)$. Let the price of the stock at time 0 be given by $\mathrm{E}\left(e^{-r T} \Phi\right)$,

$$
\Delta=\frac{\partial V}{\partial S_{0}}=\frac{\partial \mathrm{E}^{\mathrm{Q}}\left(e^{-r T} \Phi\left(S_{T}\right)\right)}{\partial S_{0}}=\mathrm{E}^{\mathrm{Q}}\left[e^{-r T} \Phi^{\prime}\left(S_{T}\right) \frac{\partial S_{t}}{\partial S_{0}}\right]=\frac{e^{-r T}}{S_{0}} \mathrm{E}^{\mathrm{Q}}\left(\Phi^{\prime}\left(S_{T}\right) S_{T}\right) .
$$

From proposition 5.5 , by letting $\mathrm{u}=1, \mathrm{~F}=\mathrm{S}_{\mathrm{T}}$, and $\mathrm{G}=\mathrm{S}_{\mathrm{T}}$, we obtain

$$
D^{u} S_{T}=\int_{0}^{T} D_{t} S_{t} d t=\int_{0}^{T} \sigma S_{T} d t=\sigma T S_{T}
$$

From the condition in the above remark, we have

$$
\delta\left(S_{T}\left(\int_{0}^{T} D_{t} S_{T} d t\right)^{-1}\right)=\delta\left(\frac{1}{\sigma T}\right)=\int_{0}^{T} \frac{d W}{\sigma T}=\frac{W_{T}}{\sigma T} .
$$

As a result,

$$
\Delta=\frac{e^{-r T}}{S_{0} \sigma T} \mathrm{E}^{Q}\left(\Phi\left(S_{T}\right) W_{T}\right) .
$$

The weight is given by

$$
\text { weight }=\frac{W_{T}}{S_{0} \sigma T} .
$$

\subsection{Computation of Gamma}

$$
\begin{aligned}
\Gamma & =\frac{\partial^{2} V_{0}}{\partial S_{0}^{2}}=\frac{\partial^{2}\left(\mathrm{E}^{Q}\left(e^{-r T} \Phi\left(S_{T}\right)\right)\right)}{\partial S_{0}^{2}}=\mathrm{E}^{Q}\left(e^{-r T} \Phi^{\prime \prime}\left(S_{T}\right)\left(\frac{\partial S_{T}}{\partial S_{0}}\right)\left(\frac{\partial S_{T}}{\partial S_{0}}\right)\right) \\
& =\mathrm{E}^{Q}\left(e^{-r T} \Phi^{\prime \prime}\left(S_{T}\right)\left(\frac{\partial S_{T}}{\partial S_{0}}\right)^{2}\right)=\frac{e^{-r T}}{S_{0}^{2}} \mathrm{E}^{Q}\left(\Phi^{\prime \prime}\left(S_{T}\right) S_{T}^{2}\right) .
\end{aligned}
$$

We now apply the Malliavin derivative property in order to eliminate ".". Suppose $\Phi^{\prime}$ is Lipschitz, let $\mathrm{G}=S_{T}^{2}, \mathrm{~F}=\mathrm{S}_{\mathrm{T}}$ and $\mathrm{u}=1$.

From proposition 5.5 we have

$$
\begin{aligned}
\delta\left(S_{T}^{2}\left(\int_{0}^{T} D_{t} S_{T} d t\right)\right)^{-1} & =\delta \frac{S_{T}}{\sigma T}=S_{T} \delta\left(\frac{1}{\sigma T}\right)-\left\langle D S_{T}, \frac{1}{\sigma T}\right\rangle \\
& =S_{T} \int_{0}^{T} \frac{1}{\sigma T} d W-\left\langle\frac{d S_{T}}{d W}, \frac{1}{\sigma T}\right\rangle \\
& =S_{T} \frac{W_{T}}{\sigma T}-\int_{0}^{T} \frac{\sigma S_{T} d t}{\sigma T} \\
& =S_{T} \frac{W_{T}}{\sigma T}-S_{T}=S_{T}\left(\frac{W_{T}}{\sigma T}-1\right) .
\end{aligned}
$$

Thus, 


$$
\mathrm{E}^{Q}\left(\Phi^{\prime \prime}\left(S_{T}\right) S_{T}^{2}\right)=\mathrm{E}^{Q}\left(\Phi^{\prime}\left(S_{T}\right) S_{T}\left(\frac{W_{T}}{\sigma T}-1\right)\right) .
$$

Using Malliavin property, we now eliminate the remaining "“".

From proposition 5.5, taking $G=S_{T}\left(\frac{W_{T}}{\sigma T}-1\right) . F=S_{\mathrm{T}}$ and $\mathrm{u}=1$ gives

$$
\begin{aligned}
\delta\left(S_{T}\left(\frac{W_{T}}{\sigma T}-1\right)\left(\int_{0}^{T} D_{t} S_{T} d t\right)^{-1}\right) & =\delta\left(S_{T}\left(\frac{W_{T}}{\sigma T}-1\right) \frac{1}{\sigma T S_{T}}\right) \\
& =\delta\left(S_{T}\left(\frac{W_{T}}{\sigma T}-1\right)\left(\sigma T S_{T}\right)^{-1}\right) \\
& =\delta\left(\frac{W_{T}}{\sigma^{2} T^{2}}-\frac{1}{\sigma T}\right)=\delta\left(\frac{W_{T}}{\sigma^{2} T^{2}}\right)-\delta\left(\frac{1}{\sigma T}\right) \\
& =W_{T} \delta\left(\frac{1}{\sigma^{2} T^{2}}\right)-\left\langle D W_{T}, \frac{1}{\sigma^{2} T^{2}}\right\rangle-\int_{0}^{T} \frac{d W}{\sigma T} \\
& =W_{T} \int_{0}^{T} \frac{d W}{\sigma^{2} T^{2}}-\int_{0}^{T} \frac{d t}{\sigma^{2} T^{2}}-\frac{W_{T}}{\sigma T} \\
& =\frac{W_{T} W_{T}}{\sigma^{2} T^{2}}-\frac{T}{\sigma^{2} T^{2}}-\frac{W_{T}}{\sigma T}=\left(\frac{W_{T}^{2}}{\sigma^{2} T^{2}}-\frac{1}{\sigma^{2} T}-\frac{W_{T}}{\sigma T}\right) .
\end{aligned}
$$

As a result,

This implies that

$$
\mathrm{E}^{Q}\left(\Phi^{\prime}\left(S_{T}\right) S_{T}\left(\frac{W_{T}}{\sigma T}-1\right)\right)=\mathrm{E}^{Q}\left(\Phi\left(S_{T}\right)\left(\frac{W_{T}^{2}}{\sigma^{2} T^{2}}-\frac{1}{\sigma^{2} T}-\frac{W_{T}}{\sigma T}\right)\right) .
$$

$$
\Gamma=\frac{e^{-r T}}{S_{0}^{2} \sigma T} \mathrm{E}^{Q}\left(\Phi\left(S_{T}\right)\left(\frac{W_{T}^{2}}{\sigma T}-\frac{1}{\sigma}-W_{T}\right)\right) .
$$

\subsection{Computation of Vega}

$$
\begin{aligned}
v & =\frac{\partial V_{0}}{\partial \sigma}=\frac{\partial \mathbf{E}^{Q}\left(e^{-r T} \Phi\left(S_{T}\right)\right)}{\partial \sigma} \\
& =\frac{\partial \mathbf{E}^{Q}\left(e^{-r T} \Phi\left(S_{0} \exp \left(\left(\mu-\frac{\sigma^{2}}{2}\right) T+\sigma W_{T}\right)\right)\right)}{\partial \sigma} \\
& =\mathbf{E}^{Q}\left(e^{-r T} \Phi^{\prime}\left(S_{T}\right) \frac{\partial S_{T}}{\partial \sigma}\right) \\
& =e^{-r T} \mathbf{E}^{Q}\left(\Phi^{\prime}\left(S_{T}\right) S_{T}\left(W_{T}-\sigma T\right)\right) .
\end{aligned}
$$

Applying proposition 5.5, let $\mathrm{G}=\mathrm{S}_{\mathrm{T}}\left(\mathrm{W}_{\mathrm{T}}-\sigma \mathrm{T}\right), \mathrm{F}=\mathrm{S}_{\mathrm{T}}$ and $u=1$ we have

$$
\begin{aligned}
\delta\left(S_{T}\left(W_{T}-\sigma T\right)\left(\int_{0}^{T} D_{t} S_{T} d t\right)^{-1}\right) & =\delta\left(\frac{S_{T}\left(W_{T}-\sigma T\right)}{\sigma T S_{T}}\right)=\delta\left(\frac{\left(W_{T}-\sigma T\right)}{\sigma T}\right) \\
& =\delta\left(\frac{W_{T}}{\sigma T}-1\right)=\delta\left(\frac{W_{T}}{\sigma T}\right)-\delta(1) \\
& =W_{T} \delta\left(\frac{1}{\sigma T}-\left\langle D W_{T}, \frac{1}{\sigma T}\right\rangle-\int_{0}^{T} d W\right) \\
& =W_{T} \int_{0}^{T} \frac{d W_{t}}{\sigma T}-\int_{0}^{T} \frac{d t}{\sigma T}-W_{T} \\
& =\frac{W_{T}^{2}}{\sigma T}-\frac{T}{\sigma T}-W_{T}=\left(\frac{W^{2} T}{\sigma T}-\frac{1}{\sigma}-W_{T}\right)
\end{aligned}
$$




$$
v=e^{-r T} \mathrm{E}^{Q}\left(\Phi\left(S_{T}\right)\left(\frac{W_{T}^{2}}{\sigma T}-\frac{1}{\sigma}-W_{T}\right)\right) .
$$

The above formulas still hold by means of an approximate procedure although the function $\Phi$ and its derivative are not Lipschitz. The important thing is that $\Phi$ should be piecewise continuous with jump discontinuities and with a linear growth.

The formulas are applicable in the case of European call option $\left(\Phi(x)=(x-\mathrm{K})^{+}\right)$ and European put option $\left(\Phi(x)=(\mathrm{K}-x)^{+}\right)$, or a digital $\left(\Phi(x)=1_{x>\mathrm{K}}\right)$.

\section{References}

[1] Bally, V., Caramellio, L. \& Lombardi, L. (2010). An introduction to Malliavin Calculus and its application to finace. Lambratoire d'analysis et de Mathématiques. Appliquées, Uviversité Paris-East, Marne-la-Vallée.

[2] El-Khatilo, Y. \& Hatemi, A.J. (2011). On the Price Sensitivities During Financial crisis. Proceedings of the World Congress on Engineering Vol I. WCE 2011, July 6-8, London, U.K

[3] Fox, C. (1950). An Introduction to Calculus of Variations. Oxford University Press.

[4] Malliavin, P. (1976). Stochastic Calculus of variations and hypoelliptic operators. In Proceedings of the International Symposium on Stochastic differential Equations (Kryto) K.Itô edt. Wiley, New York, 195-263

[5] Malliavin, P. (1997). Stochastic Analysis. Bulletin (New Series) of American Mathematical Society, 35(1), 99-104, Jan. 1998.

[6] Malliavin, P. \& Thalmaier, A. (2006). Stochastic Calculation of Variations in Mathematical Finance. Bulletin (New Series) of the American Mathematics Society, 44(3), 487-492,July 2007.

[7] Matchie, L. (2009). Malliavin Calculus and Some Applications in Finance. African Institute for Mathematical Sciences (AIMS), South Africa.

[8] Nualart, D. (2006). The Malliavin Calculus and Related Topics: Probability and its Applications. Springer $2^{\text {nd }}$ edition.

[9] Nualart, D.G. (2009). Lectures on Malliavin Calculus and its applications to Finance. University of Paris.

[10] Nunno, D.G. (2009). Introduction to Malliavin Calculus and Applications to Finance,Part I. Finance and Insurance, Stochastical Analysis and Practicalmethods. Spring School, Marie Curie.

[11] Schellhorn, H. \& Hedley, M. (2008). An Algorithm for the Pricing of Path-Dependent American options using Malliavin Calculus. Proceedings of World Congress on Engineering and Computer Science (WCECS 2008). San Francisco, U.S.A.

[12] S chröter, T. (2007). Malliavin Calculus in Finance. A Special Topic Essay.St. Hugh's College, University of Oxford. 